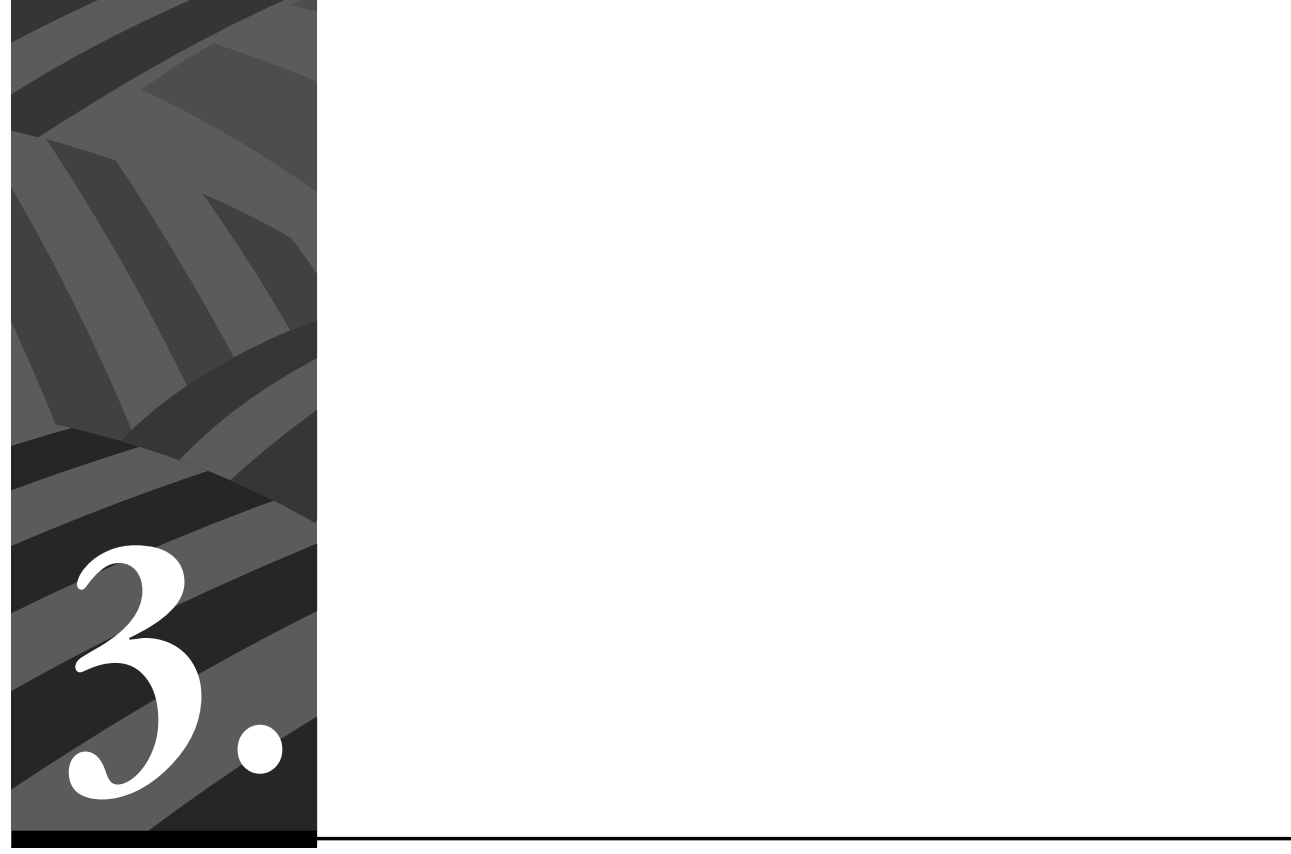

La reincorporación en clave del fortalecimiento de capacidades territoriales para la construcción de paz: una mirada desde la academia 


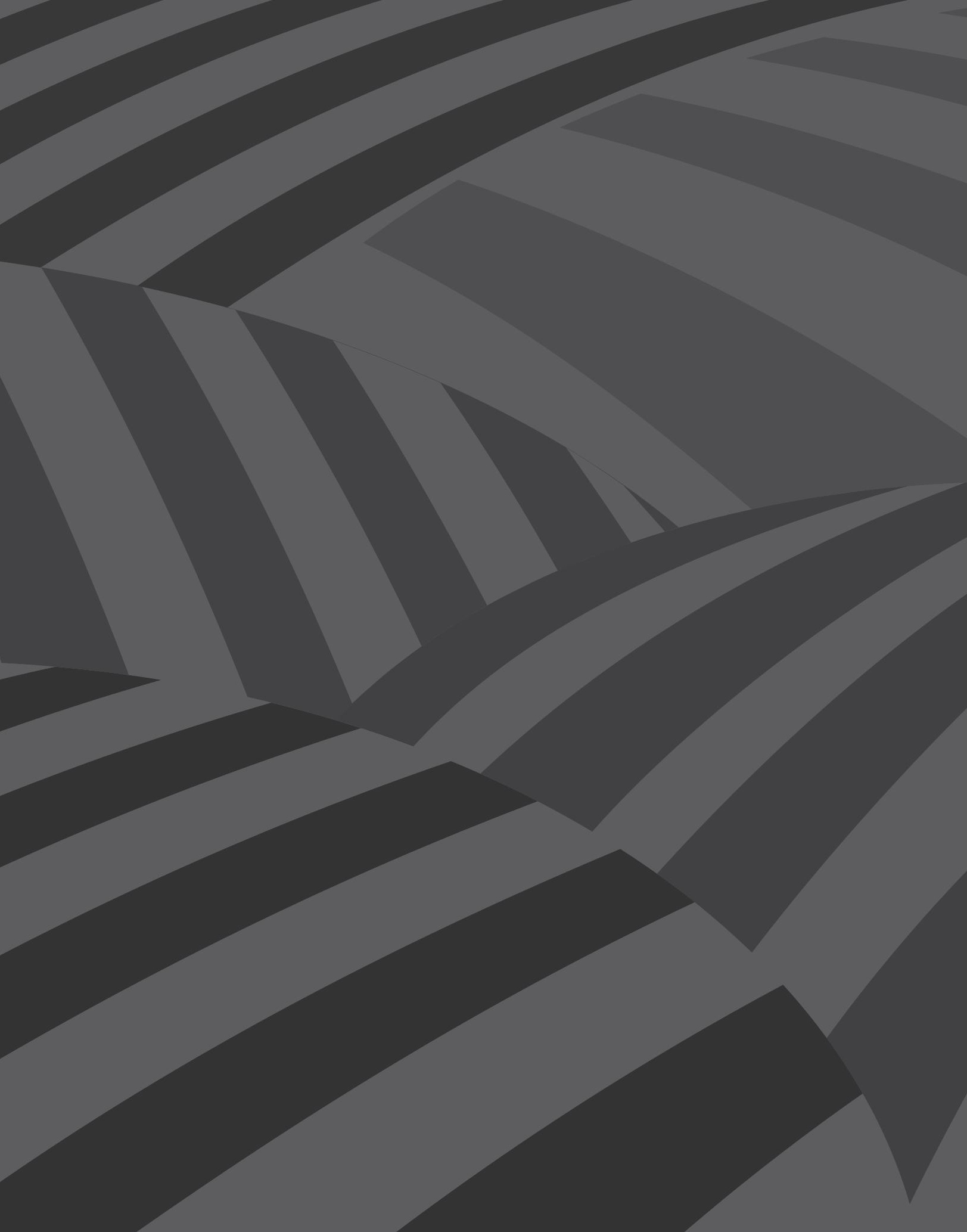




\title{
La reincorporación en clave del fortalecimiento de capacidades territoriales para la construcción de paz: una mirada desde la academia
}

\author{
Por Claudia Patricia Sierra Pardo*, María Clemencia Castro Vergara**, Jairo Ernesto \\ Luna-García $^{* * *}$, David Alejandro Fresneda Ortíz ${ }^{* * *}$, Ana Milena Muñoz Garzón ${ }^{* * * *}$
}

DOI: https://doi.org/10.54118/controver.vi217.1237

Resumen: El artículo presenta la síntesis analítica de un proceso de diálogo entre la comunidad académica de la Universidad Nacional de Colombia; los firmantes y garantes del Acuerdo de Paz en La Habana, Cuba; y representantes de las FARC-EP y del Estado colombiano alrededor de conversatorios, seguidos de un análisis del proceso de reincorporación para identificar sus alcances y limitaciones, encaminado a visualizar propuestas para su fortalecimiento en nueve ejes: comprensión de la reincorporación; enfoque territorial en la implementación de la reincorporación; acceso a la tierra; nueva geografía de la reincorporación; seguridad en los territorios y protección de la vida de personas en proceso de reincorporación; proyectos productivos, asociatividad y vínculo con procesos comunitarios; fortalecimiento de capacidades de las personas firmantes del Acuerdo; incorporación política; y nuevas dinámicas familiares, comunitarias y colectivas.

Palabras clave: Acuerdos de Paz, reincorporación, Universidad Nacional, Colombia.

* $\quad$ Profesora asociada Departamento de Trabajo Social - Facultad de Ciencias Humanas - Universidad Nacional de Colombia. Trabajadora social, magister en educación comunitaria, PhD en educación. Correo electrónico: cpsierrap@unal.edu.co

** Profesora titular Departamento de Psicología - Facultad de Ciencias Humanas Universidad Nacional de Colombia. Correo electrónico: mccastrov@unal.edu.co

*** Profesor titular adscrito a la Escuela de Diseño Industrial - Facultad de Artes Universidad Nacional de Colombia. Médico, especialista en salud ocupacional, magíster y PhD en salud pública. Correo electrónico: jelunag@unal.edu.co

**** Psicólogo de la Universidad Nacional de Colombia. Investigador del Observatorio de Paz y Conflicto. Correo electrónico: dafresnedao@unal.edu.co

***** Psicóloga de la Universidad Nacional de Colombia. Investigadora del Observatorio de Paz y Conflicto. Correo electrónico: anammunozgar@unal.edu.co 


\section{Reincorporation Process in the Sense of the Strengthening of Local Capacities for Peace- building: A view from the Academy}

Abstract: Through a dialogue between the academic community of the National University of Colombia, the signatories and guarantors of the Peace Agreement between the FARC-EP and the Colombian State signed in Havana, Cuba, an analysis of the reincorporation process was carried out in order to identify its scope and limitations. It aims at visualizing proposals for the strengthening of the agreement in nine axes: understanding of the reincorporation process; access to land; new geography of the reincorporation; security in the territories and protection of the lives of people in the process of reincorporation; productive projects, association and linkage to community processes; capacity-building of the signatories; political incorporation; and new family, community and collective dynamics.

Keywords: Peace Agreement, Reincorporation Process, National University, Colombia.

Cómo citar este artículo: Sierra Pardo, Claudia Patricia, Castro Vergara, María Clemencia, LunaGarcía, Jairo Ernesto, Fresneda Ortíz, David Alejandro y Muñoz Garzón, Ana Milena (2021). La reincorporación en clave del fortalecimiento de capacidades territoriales para la construcción de paz: una mirada desde la academia. Revista Controversia, 217, 89-119.

Fecha de recepción: 28 de febrero de 2021

Fecha de aprobación: 21 de mayo de 2021

\section{Introducción}

U

no de los temas más sensibles y relevantes dentro del acuerdo suscrito con las antiguas FARC-EP es el proceso de reincorporación a la vida civil de quienes decidieron alzarse en armas como opción para la transformación de la realidad colombiana. Fue un tema abordado al final de la negociación, con estrategias de reubicación territorial a través de las zonas veredales transitorias de normalización (ZVTN) y los puntos transitorios de normalización (PTN), luego espacios territoriales de capacitación y reincorporación (ETCR). Con el tiempo estos se agotaron por los límites de las lógicas institucionales, por el desespero de quienes habiendo firmado la paz no vieron la implementación prometida y salieron de allí buscando otras alternativas, o por la acción de actores armados que presionaron la reubicación de algunos de estos ETCR. 
Considerando la relevancia de este proceso y los impactos determinantes que su desarrollo tendrá sobre la construcción de paz en nuestro país, se identificó el tema de la reincorporación como centro de una indagación realizada por un grupo de docentes y estudiantes, a través de la conformación del Foco de Pensamiento denominado "Aporte de la Universidad Nacional de Colombia a los ETCR, las nuevas áreas de reincorporación (NAR) y el fortalecimiento de capacidades locales para la paz territorial". La iniciativa estuvo apoyada por la Vicerrectoría de Investigación y Extensión de la sede Bogotá, en el marco de la Escuela de Pensamiento Universitario.

La estrategia Focos de Pensamiento Universitario hace parte de esta Escuela y busca contribuir al análisis y propuestas sobre temas neurálgicos para el país, aportando a la cualificación o formulación de acciones de política pública. Desde la iniciativa propuesta en relación con el proceso de reincorporación en desarrollo, interesó escuchar las voces de los distintos actores involucrados: Gobierno, personas integrantes de las antiguas FARC-EP y entidades de la cooperación internacional vinculadas a la verificación de lo pactado. Con esta intención se realizaron cuatro conversatorios en el mes de agosto del año 2020 en los que participaron: Emilio José Archila Peñalosa, Alto Consejero Presidencial para la Estabilización y la Consolidación; Pastor Alape y Consuelo Fuertes, delegados por el Partido FARC ante el Consejo Nacional de Reincorporación; Jesús Emilio Carvajalino, integrante de la Junta Directiva de Corpo-Reconciliación ${ }^{1}$; y Alessandro Preti, jefe de Reincorporación de la Misión de Verificación de la ONU en Colombia.

La metodología adelantada implicó, en un primer momento, la escucha independiente de cada actor para comprender de mejor forma sus análisis, posturas y valoraciones respecto al camino recorrido hasta ahora. Posteriormente, se procedió a la realización de los conversatorios y la

1 Organización no gubernamental integrada por alrededor de 2000 excombatientes de las FARC, conformada con el fin de agrupar a personas no incorporadas en el nuevo partido. 
reconstrucción de cada uno de ellos mediante una relatoría detallada; y, un tercer momento correspondió a la elaboración de una síntesis analítica, a partir de la cual se construyó el presente artículo.

Los conversatorios permitieron identificar varios ejes temáticos relevantes en el desarrollo del proceso de reincorporación. Estos fueron analizados desde lo planteado por cada uno de los actores participantes, identificando tensiones, limitantes, asuntos por resolver, potencialidades y, sobre todo, acciones, propuestas e iniciativas posibles desde la Universidad Nacional de Colombia para aportar al proceso de reincorporación orientado al fortalecimiento de capacidades territoriales para la construcción de paz.

El presente artículo retoma los resultados de la indagación realizada con base en los conversatorios y su organización en nueve ejes temáticos respecto a asuntos fundamentales dentro del proceso de reincorporación: comprensión de la reincorporación; enfoque territorial en la implementación de la reincorporación; acceso a la tierra; nueva geografía de la reincorporación; seguridad en los territorios y protección de la vida de personas en proceso de reincorporación; proyectos productivos, asociatividad y vínculo con procesos comunitarios; fortalecimiento de capacidades de las personas firmantes del Acuerdo; incorporación política; y nuevas dinámicas familiares, comunitarias y colectivas. Sobre cada eje se presenta una síntesis de lo planteado en una perspectiva analítica y, al final de estos, algunas propuestas o iniciativas universitarias que van perfilando líneas de acción posibles para continuar el aporte institucional en estos temas.

\section{Eje 1. Comprensión de la reincorporación}

"De acuerdo con tu comprensión de lo que ves, actúas..."2.

Al analizar los procesos de implementación de lo pactado, un elemento esencial son las concepciones sobre asuntos fundamentales relativos

2 Aporte de profesora participante en el conversatorio del 12 de agosto de 2020. 
a los contenidos del Acuerdo. Se pueden citar algunos ejemplos: cómo se comprende el origen mismo del conflicto, o la reforma rural integral, o la reincorporación. Parece ser que, desde la etapa de negociaciones públicas en La Habana, el asunto de la reincorporación se concibió como un capítulo corto, abordado y escrito en la instancia final del proceso, construido con la pretensión de ir más allá de un conjunto de beneficios para otorgar a quienes decidían salir de la guerra, y con la expectativa de una articulación orgánica a las demás partes del Acuerdo. Sin embargo, el desarrollo del proceso hasta la fecha muestra la existencia de distintas comprensiones respecto a la reincorporación que han generado tensiones. Se enuncian a continuación las cuatro más visibles, a manera de pregunta:

\section{1 ¿Beneficiarios o sujetos políticos?}

Esta tensión se hace visible en la percepción de integrantes de las antiguas FARC-EP, en el sentido de ser tratados y vistos como destinatarios de "paquetes de beneficios" o como simples receptores de la acción decidida y pensada por terceros en el escenario institucional que antes impugnaron y quisieron transformar. Esta concepción anula su capacidad de decisión y de agencia, y además los invisibiliza como actores relevantes en la construcción de la paz, reforzando la idea de un papel protagónico solo en la guerra.

Contrario a ello, quienes han firmado el Acuerdo siendo integrantes de las antiguas FARC-EP, se reconocen a sí mismos como sujetos, esto es, con un proyecto a futuro, capacidad de acción para construirlo y compromiso en hacerlo. Son sujetos con derechos, potencialidades y deseo de aportar activamente a la construcción de la paz en el país.

Este es un asunto relevante en el análisis del proceso de reincorporación adelantado hasta ahora, el cual puede rastrearse también en la forma como se denomina y autodenomina a este colectivo, porque las formas de nombrar o nombrarse son indicativas también de la concepción del 
proceso y marcan tensiones entre las lógicas de la institucionalidad y las reivindicaciones políticas de quienes transitan a la vida civil: firmantes del Acuerdo, excombatientes, personas en proceso de reincorporación (PPR), exintegrantes, reincorporados, etc. Desde el comienzo de la implementación, términos como excombatiente o exguerrillero/a, son objeto de debate, puesto que algunas de estas personas no se reconocen en la expresión que señala el 'dejar de ser’ —algo que se extingue-, sino en una identidad que se transforma y opera por vías alternas. Por eso, el sentido que se reivindica está asociado a la vigencia de lo que se ha sido y por ello no se asume la expresión "ex" combatiente, sino su afirmación: "es combatiente".

Analizar las formas de nombrar al otro/a la otra en el relacionamiento que los distintos actores involucrados realizan con quienes hacen tránsito a la vida civil, es un tema fundamental, aunque no lo pareciera. Además, tiene un efecto en la opinión pública del país, pues construir nuevos lenguajes y nuevas formas de nombrar hace parte también de los procesos imprescindibles de emprender para lograr avanzar hacia la reconciliación.

\section{2 ¿La integración económica es el centro de la reincorporación?}

Desde la perspectiva gubernamental, uno de los principales logros del proceso de reincorporación es la vinculación de los integrantes de las antiguas FARC-EP al sistema financiero (bancarización) en un $98 \%$, al de salud en un $85 \%$, así como otros avances identificados por el Gobierno nacional desde la lógica productiva. El aspecto económico es relevante cuando se trata de ofrecer alternativas de ingresos distintas a las de la guerra y sus lógicas, y se valora como un mecanismo de contención de la vuelta al uso de las armas. De allí su centralidad en el proceso de reincorporación.

Pero, desde una perspectiva de integralidad, se insiste en la relevancia de concebir la reincorporación como un proceso complejo, con centro 
múltiple, cuyo éxito pasa por estos asuntos sin que ellos sean el único o más exitoso componente. Desde esta última perspectiva, asuntos como el acceso a una vivienda digna, la participación activa de exintegrantes de las FARC-EP en nuevas formas de configuración y organización territorial, y el reconocimiento de nuevas áreas de reincorporación urbanas, además de los ETCR, son altamente importantes. Sin embargo, a la fecha no son reconocidos como tal en la lógica institucional e incluso se puede afirmar que hay un avance muy limitado en la implementación de los acuerdos del punto 1: Reforma Rural Integral (Naranjo, Machuca y Valencia, 2020). La pregunta que queda planteada para la reflexión con respecto a esta tensión sería, ¿cómo lograr un proceso de reincorporación verdaderamente integral, con efectos positivos en las distintas dimensiones de la vida personal y colectiva de quienes transitan a la vida civil?

\section{3 ¿Reincorporación o reintegración?}

Para quienes suscribieron el Acuerdo siendo integrantes de las antiguas FARC-EP, la institucionalidad debe diferenciar los procesos de reintegración y los de reincorporación y, en virtud de ello, evitar comprender el proceso en curso como expresión del sometimiento de las ciudadanas y los ciudadanos que suscribieron el Acuerdo de Paz (Zambrano, 2019).

Si bien tanto Naciones Unidas como el Gobierno colombiano han hablado de reintegración - $-\mathrm{y}$, en el caso de nuestro país, podemos recordar la experiencia de la Agencia Colombiana para la Reintegración-, el concepto evoca, teóricamente, la concepción de paz negativa expresada por Johan Galtung, como ausencia o reducción de violencia directa (2003, p. 31). La experiencia de países como Colombia, con conflictos armados de décadas, ha mostrado que esta perspectiva se queda corta y que la transición a la vida civil de quienes deciden dejar las armas es un proceso mucho más allá del no uso de estas.

En el otro eje de esta tensión se encuentran las posturas que llaman a la reincorporación, término que permite reconocer múltiples aspectos del 
tránsito a la vida civil, tras la dejación de armas. En este sentido, alude mucho más a la concepción de paz positiva, entendida como transformación creativa y no violenta de los conflictos (Galtung, 2003, p. 31). Reconocer y resolver esta tensión en favor de una concepción integral del proceso es fundamental para avanzar por caminos de no repetición, los cuales permitan romper verdaderamente las lógicas que perpetúan la guerra.

\section{4 ¿Integralidad vs. fragmentación, o la tensión entre lo colectivo y lo individual y?}

Al margen de las cifras con las que se mostraron avances y estancamientos en el proceso de reincorporación, se plantean valoraciones sobre lo que han generado las estrategias puestas en desarrollo hasta ahora. La conclusión común entre distintos integrantes de las antiguas FARC-EP fue la misma: fragmentación. La sensación de incumplimiento, también recurrente, se acompaña de esfuerzos constantes y variados por superar la individualización a la que, pareciera, se les quiere llevar en la transición a la vida civil. Las estrategias desarrolladas para la reincorporación no están fortaleciendo las capacidades locales para la paz, presentes en la antigua insurgencia como colectivo. Las mayores demoras, requisitos y obstáculos que registran los proyectos productivos de corte colectivo son, en algunos casos, pruebas de ello, así como las visibles diferencias en el número de propuestas aprobadas de uno y otro nivel ${ }^{3}$. Aquí aparece un elemento relevante: la idea de colectividad. Quienes pertenecieron a las antiguas FARC-EP se entendieron, durante décadas, como colectivo no solo por la unidad de mando o la disciplina al interior de la organización insurgente, sino por la comprensión de estar comprometidos en un proyecto de vida común. Este factor es fundamental y es, en buena parte, el eje que se encuentra en la base de esta tensión.

3 Hasta agosto de 2020, ha sido aprobado un total de 60 proyectos productivos colectivos, que involucran a 2800 personas y 1400 iniciativas individuales, que benefician a 1600 personas, según cifras proporcionadas por la Alta Consejería Presidencial para la Estabilización y Consolidación (ACPEC). 
Pareciera que los actores gubernamentales temen que, al fortalecer la idea del colectivo organizado, pueda mantenerse vivo el riesgo de una vuelta a la opción armada, postura comprensible pero no favorable para una reincorporación sólida. En perspectiva contraria a este temor, integrantes de las antiguas FARC-EP insisten en haber logrado apropiarse de la reincorporación, situación que se evidencia en la decisión de mantener su compromiso con lo pactado, incluso por encima de los incumplimientos registrados, pero, eso sí, desde iniciativas que favorezcan la unión de los excombatientes.

Como un tema transversal en el proceso de reincorporación, surge el debate y la apuesta por la colectividad, insistiendo en la necesidad de superar el enfoque individualista que la institucionalidad está privilegiando, sobre el enfoque colectivo pactado en el Acuerdo Final de Paz. Se necesita pensar estrategias que favorezcan los proyectos colectivos, incluso para personas que se encuentran fuera de los ETCR, en nuevas áreas de vivienda o con sus familias de origen, y que requieren de un referente y pertenencia existencial. Esto también les permitiría mantenerse fieles al compromiso con la paz.

Lo que queda claro en relación con la comprensión del proceso de reincorporación

Desde el análisis planteado por los distintos actores, el proceso de reincorporación de las antiguas FARC-EP se formuló y consignó en los documentos correspondientes, en una perspectiva integral, sostenible y amplia. Sin embargo, en algunos de sus desarrollos se acerca más a la lógica de la reintegración: un proceso individual de anclaje en la vida social y en la dinámica productiva y financiera del país, sin implicaciones políticas. Por esta vía, las personas involucradas son vistas como beneficiarios, no como sujetos con capacidad de agencia, decisión y protagonismo, y el eje de la acción institucional pareciera ser meramente económico o técnico-productivo; por lo menos así es percibido por las personas involucradas. 
Ha sido posible identificar también las persistencias en el proceso de reincorporación, evidentes en las palabras de algunos participantes que indicaron, por ejemplo, el estar "aferrados a la reincorporación” o verla como "una tabla de salvación”; o en reconocer el compromiso por parte de los excombatientes "con el proceso pactado en La Habana, con la reconciliación y la reincorporación”. La lectura gubernamental coincide en este punto al señalar que "aún con las amenazas e invitaciones de quienes nunca dejaron el narcotráfico, muy pocos excombatientes han salido del proceso". El compromiso con y la apropiación de la reincorporación por parte de los integrantes de las antiguas FARC-EP se hace evidente, también, en la decisión de conformar una familia con hijos e hijas. Este tipo de decisiones denota deseo y búsqueda de estabilidad.

El tipo de proyectos e iniciativas que como Universidad Nacional de Colombia venimos adelantando con respecto a la reincorporación, muestran que institucionalmente hemos respaldado los procesos conducentes a lo que se conoce como peacemaking (hacer la paz), asociados a la negociación con las insurgencias específicamente (paz negativa). Nuestro aporte institucional se ha orientado, también, hacia el sostenimiento de la paz (peacekeeping) y, fundamentalmente, hacia su construcción (peacebuilding). Es decir, en la concepción del proceso de reincorporación que evidenciamos al interior de la Universidad, se identifica una distancia respecto a los lineamientos clásicos para pensar la paz, sobre el entendido que la firma del Acuerdo con las FARC-EP, en 2016, debe aportar al país condiciones para una paz positiva perdurable en el tiempo, que garantice estabilidad en un proceso de largo aliento y, en consecuencia, debe priorizar su construcción en perspectiva territorial. La Universidad Nacional de Colombia puede y debe continuar aportando a la construcción de un proceso de reincorporación integral, que fortalezca las capacidades para la paz y la transformación de conflictos tanto en las comunidades de los territorios afectados por la guerra como en los colectivos y actores involucrados. 


\section{Eje 2. Enfoque territorial en la implementación de la reincorporación}

"El enfoque territorial debe vincular los procesos de reincorporación con la transformación misma de los territorios, armonizando el desarrollo de escenarios como los ETCR y las NAR, con la ejecución de los PDET, el PNIS, los planes de desarrollo municipales y departamentales, y los instrumentos de ordenamiento territorial"4.

El segundo eje temático respecto al cual se produjeron reflexiones en los conversatorios sitúa el proceso de reincorporación de cara a su propósito mayor: la construcción de paz en un país resquebrajado por el conflicto armado. Este objetivo da sentido a la reincorporación por encima de lo que compete exclusivamente a integrantes de las antiguas FARC-EP, pues involucra distintos escenarios institucionales y territoriales, y, así mismo, un conjunto amplio de actores y sectores sociales con roles diferenciados tanto en la apuesta por la paz como en el proceso de la reincorporación. Es decir, esta última tiene una dimensión territorial que supera la figura de los antiguos ETCR o nuevas expresiones de agrupamiento de personas en proceso de reincorporación.

Todos los actores coincidieron en la presencia de un enfoque territorial en la reincorporación, pero con énfasis distintos. Por un lado, el trabajo de la Agencia de Renovación del Territorio y la Agencia para la Reincorporación y la Normalización en la articulación de los Programas de Desarrollo con Enfoque Territorial (PDET) con los planes de desarrollo de los municipios priorizados, pasando por la activación y funcionamiento de 24 Consejos Territoriales de Reincorporación, hasta llegar a espacios de concertación como 12 mesas departamentales de reincorporación activas a agosto del año 2020; todo ello valorado como logros del enfoque. En relación con la construcción y desarrollo de los PDET, el Partido

4 Alessandro Preti, conversatorio 12 de agosto de 2020. 
FARC evidenció su incapacidad para acompañar este proceso, dado que no cuenta con recursos para ello.

La reincorporación fue vinculada a escenarios e instancias asociadas con la planeación del desarrollo local y la construcción de política pública en el ámbito municipal, departamental e incluso distrital, con algunas experiencias productivas como la de Frutos de Paz, que se desarrolla actualmente en Bogotá.

Si bien el tema del desarrollo local es uno de los centrales dentro del enfoque territorial, en dos conversatorios se mencionaron avances escasos en políticas para impulsarlo y la urgencia en la implementación de la reforma rural integral, entendiendo el peso que este aspecto tuvo y los obstáculos desde la institucionalidad para llevarlo a cabo, así como el trabajo de fortalecimiento de las capacidades territoriales para la paz.

Una tercera línea que visibiliza el enfoque se encuentra en proyectos productivos de carácter cooperativo que trabajan en la articulación de población excombatiente con población campesina, afro, víctimas e indígenas, como la Cooperativa Agropaz. En estos casos, el enfoque territorial de la reincorporación se expresa en la vinculación de pobladores y comunidades cercanas a los ETCR, en zonas y regiones afectadas por el conflicto armado, como experiencias de trabajo conjunto que aportan a la construcción de paz territorial. Los ETCR han permitido la interacción con las comunidades cercanas y el establecimiento de relaciones de mutuo apoyo y beneficio, que en ocasiones han contribuido a procesos de reconciliación y reconocimiento entre personas distintas a las que están en proceso de reincorporación.

En relación con este segundo eje temático identificado como transversal a los cuatro conversatorios adelantados, es claro que la reincorporación tiene una contribución a la transformación de los territorios 
y a la planeación local participativa, enfatizando en experiencias de diálogo y acción conjunta con actores locales.

La presencia de la Universidad Nacional de Colombia en territorios donde confluyen personas en proceso de reincorporación con población afectada por el conflicto armado puede ser muy importante para gestar o fortalecer espacios de encuentro, interacción, diálogo y acción conjunta. La oferta educativa o de capacitación que realice la Universidad debe contribuir a estas formas de relación, para fortalecer la construcción de paz desde el modelo local solidario que presupone un proceso que va de "abajo hacia arriba", en el cual la autonomía de los territorios es un valor por preservar y un mínimo ético de las acciones sin daño.

\section{Eje 3. Acceso a la tierra}

"La tierra es un tema básico para la sostenibilidad del proceso de reincorporación"s.

La alusión al acceso a la tierra como elemento fundamental para la consolidación de la reincorporación se plantea en dos sentidos. Por un lado, como factor que articula orgánicamente la transición a la vida civil con la comprensión, necesaria y urgente, de la reforma rural integral incluida en el Acuerdo Final, como un punto básico para avanzar verdaderamente hacia la paz. En segundo lugar, como otro de los factores que actualmente genera mayores tensiones e inquietudes respecto al futuro de la reincorporación.

Desarrollando el primer sentido con el cual aparece el acceso a la tierra en la discusión sobre la reincorporación, se reiteró que el carácter político del debate sobre la tierra dificulta un abordaje más claro por parte de la institucionalidad. Sin lugar a duda la tierra y el acceso a esta sigue siendo centro de debate y asunto básico en la transición a la paz, así como fue

5 Alessandro Preti, conversatorio 12 de agosto de 2020. 
y sigue siendo detonante del conflicto armado en sí mismo. Garantizar el acceso a la tierra y la puesta en marcha de una reforma rural integral - siguiendo lo pactado-, es visto por varios actores como camino real de transformación territorial, pues sus avances permitirían materializar la consolidación de la paz y del proceso mismo de reincorporación.

En relación con el segundo sentido, habiendo concebido desde el inicio los ETCR como espacios transitorios, llegando al final de esta estrategia territorial gana relevancia el tema de la adquisición de predios. Para integrantes de las antiguas FARC-EP, la reincorporación integral implica también la compra de tierras que hagan viable el desarrollo de proyectos productivos y de vivienda, en condiciones de sostenibilidad y seguridad. Se plantea como evidente la ausencia de un plan de vivienda para la reincorporación tanto en áreas rurales como urbanas, y una falta de interés del Gobierno nacional por formalizar una ruta para lograr el acceso a tierras en la reincorporación. Esto ha llevado a un trabajo del componente FARC en el Consejo Nacional de Reincorporación (CNR), enfocado a la articulación con otros entes involucrados en buscar esta viabilidad.

Desde la perspectiva de la institucionalidad se mencionaron los procesos ya iniciados de compra de predios, orientados al desmonte concertado de algunos AETCR, manteniendo la presencia de excombatientes en ciertos territorios donde estos se asentaron. En relación con ello se resaltó el que autoridades municipales manifiesten el deseo de permanencia de esta población en el territorio, quizás en primera instancia por la mayor posibilidad de inversión de recursos públicos que les representa. Y, de otra parte, el hecho de que la compra de terrenos para la reincorporación no quedó contemplada en el Acuerdo Final de Paz y, por lo tanto, estas adquisiciones no han tenido lugar. Sin embargo, pareciera vislumbrarse ya una ruta jurídica, con algunos desarrollos normativos que dan la posibilidad de incluir predios de la Sociedad de Activos Especiales, o la posibilidad, aún en revisión, de utilizar el recurso de los 8 millones que se otorgan a proyectos productivos, también para el tema de tierras. 
El asunto del acceso a la vivienda genera tensión, dado que es visto por la institucionalidad como un tema que, a pesar de no estar contemplado en el Acuerdo, ha sido abordado por el Gobierno nacional en la hoja de ruta de la reincorporación como uno de los aspectos centrales del proceso, contrario a la opinión de los firmantes del Acuerdo.

Los planteamientos registrados sobre el tema de acceso a tierras en los conversatorios muestran la ausencia de un abordaje estratégico desde el punto de vista de las personas en proceso de reincorporación, especialmente si se encuentran fuera de los ETCR. Esto tiene que ver con el carácter político de las discusiones alrededor del tema. Aunque no se cite explícitamente en el Acuerdo Final, allí sí se menciona la necesidad de garantizar la integralidad de los procesos de reincorporación. Pero un proceso integral y a largo plazo demanda la creación de un plan de acción definido, con un alcance mayor que el apoyo económico puntual por proyecto, así como mecanismos concretos que garanticen de forma efectiva el acceder a tierra, crédito, mercado, asistencia técnica y fortalecimiento organizativo.

Con esto, es evidente también que el panorama de acceso a tierras es aún más difuso para quienes se encuentran fuera de los ETCR, pues las diferentes y numerosas formas de asentamiento donde se ha llevado a cabo la reincorporación en entornos rurales o urbanos no fueron contemplados, en principio, por el Acuerdo suscrito.

La garantía del acceso a la tierra es eje fundamental en la consolidación de la reincorporación y elemento constitutivo para lograr el anclaje a territorios concretos de la población en proceso de retorno a la vida civil, dada además su alta vocación hacia lo agropecuario. Igual sucede con propósitos fundamentales como el fortalecimiento de los vínculos sociales, tan necesarios para procesos de reconciliación. De otra parte, la tierra es indispensable para el desarrollo de los proyectos productivos propuestos y la satisfacción de necesidades de vida, dentro de las que requiere particular atención el acceso a una vivienda digna. 


\section{Eje 4. Nueva geografía de la reincorporación}

"Ha evolucionado la geografía de la reincorporación y desde el 2019 nos encontramos en una nueva etapa denominada de consolidación, en la cual es clave el arraigo. Pero ¿arraigo a qué y cómo lograrlo? Porque nuestro proceso de reincorporación ya no está [solamente] en los ETCR"6.

La movilidad geográfica de los excombatientes después de su concentración en las ZVTN y los posteriores ETCR, fue uno de los factores que no se consideró en las negociaciones del Acuerdo Final. Si bien inicialmente los más de 13000 firmantes del Acuerdo se concentraron en estos lugares, con el paso del tiempo el número fue disminuyendo hasta llegar, en agosto de 2020, a menos de 3000 personas en ellos. ¿Qué generó esta situación? Algunos de los participantes en los conversatorios plantearon que, en la etapa de reincorporación temprana, el Gobierno no había terminado la adecuación infraestructural de los lugares, ni respondió oportunamente demandas al respecto. Esta fue una de las primeras situaciones que con el tiempo generaron desplazamientos de firmantes del Acuerdo hacia otros ETCR e incluso hacia zonas urbanas en distintas ciudades, incluida Bogotá. Otras razones se relacionan con la cercanía a familiares, la búsqueda de condiciones óptimas para desarrollar proyectos productivos, o la inseguridad asociada a amenazas y asesinatos, una realidad presente en los ETCR y en otras regiones del país.

De acuerdo con integrantes del componente FARC, en el CNR se tienen identificadas actualmente entre 80 y 90 nuevas áreas de reincorporación en el territorio nacional; la Misión de Verificación de la ONU, a agosto de 2020, había ubicado 75 asentamientos. Se sortean dificultades para mantener comunicación con firmantes del Acuerdo allí localizados, debido a la ausencia de enlaces territoriales, a pesar de que varias de las NAR están articuladas regionalmente con los ETCR. Según se planteó

6 Alessandro Preti, conversatorio 12 de agosto de 2020. 
"las NAR son colectivos que se identifican por sus acciones productivas de manera colectiva, pero no viven en colectivo" .

\subsection{Dinámicas colectivas de reincorporación}

Esta nueva geografía de la reincorporación se problematiza con la posición institucional, la cual no reconoce a las NAR como áreas de reincorporación, dado que se salen del marco legal previsto. En consecuencia, aquellos excombatientes que se desplazan fuera de los ETCR están “creándole un problema al proceso”, pues los lugares seleccionados por ellos no siempre se encuentran en las zonas destinadas para construcción de vivienda, según el ordenamiento territorial de los municipios. Desde la mirada institucional no se reconocen espacios de reincorporación fuera de los AETCR, con lo cual es difícil apoyar a la población en reincorporación en temas como vivienda, salud y educación, generándose una situación de inequidad entre quienes habitan estos escenarios y quienes están fuera de ellos. A pesar de esta ausencia de reconocimiento, se afirma que "el proceso de reincorporación no está de ninguna manera limitado a quienes están en los AETCR”, ya que el compromiso adquirido en el Acuerdo es con la totalidad de ellos.

En relación con este eje temático quedan planteadas dos inquietudes por ahora. Una de ellas respecto al futuro de los AETCR y la posibilidad de su transición a otras expresiones territoriales, como poblados rurales o poblados de paz. Esto como respuesta al deseo de quienes deciden permanecer allí para generar o fortalecer su arraigo, encontrando alguna figura jurídica que lo haga posible. Así se avanzaría con el análisis realizado por el Gobierno sobre las necesidades para la consolidación de los espacios a mantener (según decisión de los firmantes del Acuerdo) y con la adquisición de predios nuevos, en los casos de traslado. En algunos lugares se

7 Al respecto, véase Misión de Verificación de las Naciones Unidas en Colombia, 2020. 
contaría con el respaldo de las administraciones y comunidades locales para este propósito.

La segunda inquietud está ligada al reconocimiento de los espacios urbanos en distintas ciudades del país como escenarios de reincorporación. En uno de los conversatorios se planteó, por ejemplo, que en Bogotá se han ubicado más de 900 excombatientes, quienes siguen adelante con su proceso de reincorporación a pesar del no reconocimiento por parte del Gobierno. La reincorporación urbana demanda acciones institucionales enfocadas a sus características particulares, de la misma forma que los enfoques territorial y diferencial del Acuerdo han guiado los esfuerzos alrededor de los ETCR.

\section{Eje 5. Seguridad en los territorios y protección de la vida de personas en proceso de reincorporación}

"No se puede hablar de reincorporación económica y social si no hay condiciones politicas en el territorio que garanticen la vida y la seguridad de los firmantes del Acuerdo".

"Proteger a los excombatientes debería ser un propósito de toda la sociedad y avanzar en ese tema no debería separarnos".

En la transición desde escenarios de conflicto a compromisos con la construcción de paz, se observa como una constante en experiencias del ámbito internacional la agudización de la violencia y el intento de exterminio del "enemigo político", quien al firmar la paz pareciera haberse "entregado" o "rendido”. Esta es una constatación dolorosa en el período posterior a la firma del Acuerdo de Paz con las FARC-EP en Colombia. De ahí que el tema de la seguridad fue asunto reiterado en la mayoría de los conversatorios adelantados.

8 Pastor Alape, conversatorio agosto 11 de 2020.

9 Aporte de profesora participante en el conversatorio del 11 de agosto de 2020. 
En relación con la seguridad en los territorios y la protección de la vida de los firmantes del Acuerdo de Paz, se identificaron dos conclusiones recurrentes: por un lado, la ausencia de condiciones que garanticen la integridad física, e incluso la seguridad jurídica, de las personas en proceso de reincorporación. En segunda instancia, la necesidad de establecer medidas para mitigar las acciones violentas que se están presentando en su contra.

Para todos los actores participantes en los conversatorios, es evidente la inseguridad física de los excombatientes e incluso la presencia de "discursos de odio y estigmatización” hacia las y los firmantes del Acuerdo, lo cual aumenta el riesgo y su situación de vulnerabilidad.

No concuerdan los análisis que tanto integrantes de las antiguas FARCEP como la institucionalidad han realizado sobre los responsables detrás de los asesinatos y amenazas a excombatientes firmantes del Acuerdo. La Unidad Especial de Investigación para el Desmantelamiento de Organizaciones y Conductas Criminales de la Fiscalía y otras entidades sindican a las estructuras del narcotráfico, sin que dichas acciones estén relacionadas con la condición de víctima que pueden tener los excombatientes. De otra parte, se señala a sectores que no legitiman el proceso de paz y buscan exterminar a personas en reincorporación o generar temor, inacción y silencio entre ellos y ellas. Al mismo tiempo buscan afectar el desarrollo de los proyectos y procesos en curso, aniquilando a la dirigencia más cualificada y de mayor experiencia organizativa.

Se puso sobre la mesa la ausencia de medidas para frenar las acciones en contra de excombatientes. Aunque la institucionalidad se muestra preocupada respecto a la seguridad jurídica y la garantía de su vida, esta preocupación no se traduce en acciones efectivas. Se ha llegado a una situación en la cual "quienes están siendo víctimas de la fusilería del enemigo” son sobre todo los excombatientes en territorios rurales, 
no en las grandes ciudades. Se percibe por esta vía un escalamiento de la situación de violencia y conflicto sin freno posible a la vista.

\section{Eje 6. Proyectos productivos, asociatividad y vínculo con procesos comunitarios}

Por la centralidad que tiene el componente económico de la reincorporación, el tema de los proyectos productivos se constituyó en uno de los ejes relevantes del proceso en la actualidad y en un asunto recurrente en los conversatorios realizados. El análisis planteado al respecto se fundamentó en información e indicadores cuantitativos que muestran el avance o estancamiento en la implementación de lo acordado en La Habana sobre este tema.

Se resalta el número de proyectos aprobados a la fecha, 60 en total, y de las personas en proceso de reincorporación cobijadas por las distintas iniciativas. Se destacó también la agilidad creciente en la aprobación de las propuestas durante los últimos meses, contando para ello con el apoyo de entidades nacionales como la Agencia de Emprendimiento e Innovación iNNpulsa y otras internacionales entre las que figuran el Programa de las Naciones Unidas para el Desarrollo o el Fondo Europeo para la Paz, el cual ha sido fundamental no solo para la financiación de los proyectos, sino para su correcta formulación desde el punto de vista técnico y cualitativo.

Cruzando este eje temático productivo con la lectura geográfica de la reincorporación, se tiene que, de los 60 proyectos aprobados por el CNR, alrededor del $40 \%$ se encuentran fuera de los AETCR. Esto corresponde a 3200 de las 4400 personas vinculadas a proyectos productivos, según las estadísticas para julio de 2020. Como se ha mencionado, la ausencia 
de reconocimiento de las NAR impide el apoyo institucional en este tipo de asuntos ${ }^{10}$.

De otro lado, tanto la Misión de la OnU como la Alta Consejería para la Estabilización y la Consolidación dan cuenta de 1400 iniciativas individuales que benefician alrededor de 1640 personas, haciendo énfasis justamente en la imparcialidad del Gobierno respecto al impulso de proyectos colectivos o individuales, sin dejar de anotar que 'la decisión debe ser individual'.

Para el caso de proyectos desarrollados en los contextos urbanos donde se han asentado personas en proceso de reincorporación, el Gobierno destaca el mantenimiento de las garantías para quienes se encuentran en ciudades como Bogotá o Medellín. Esto incluye la interlocución constante con instancias como la Alcaldía Distrital o la Cámara de Comercio, lo cual ha derivado no solo en el avance de los proyectos, sino en el desarrollo de nuevas propuestas como el PDET de Sumapaz.

Por su parte, desde el CNR se señaló que el éxito del proceso de reincorporación no se puede medir por el número de proyectos aprobados o los índices de rendimiento o productividad de estos. La reflexión sobre los proyectos productivos planteados por personas firmantes del Acuerdo, ya sea desde el CNR o desde Corpo-Reconciliación, parte de nociones más integrales y sistémicas de la reincorporación y de su vínculo con la implementación del Acuerdo en general. La interdependencia con el avance en temas de educación, formación y capacitación; adquisición y formalización de los predios; consolidación de la vivienda en medio del desmonte de los antiguos ETCR, entre otros, resulta clave para identificar los

10 Se hace referencia aquí a cifras mencionadas en los conversatorios, pero este es un aspecto en continuo cambio. Al respecto se pueden consultar estadísticas oficiales en la Agencia para la Reincorporación y la Normalización. 
verdaderos logros del proceso y de las gestiones institucionales. De ahí el planteamiento de una visión más crítica y política del contexto actual.

Específicamente, alrededor de lo productivo se identificaron dos aspectos fundamentales: por un lado, la asociatividad, evidente en las experiencias en desarrollo en Bogotá, como ciudad-nodo para la comercialización de lo producido en iniciativas regionales. Por otro, los problemas en la adquisición de tierras para realizar los proyectos productivos de manera adecuada. Al respecto, los y las representantes de Corpo-Reconciliación plantearon la necesidad de una descentralización de todos los componentes de la reincorporación, de tal forma que los territorios donde el proceso productivo tenga lugar se tomen en cuenta bajo sus condiciones particulares, de modo que puedan adquirir mayor autonomía en sus decisiones.

En cuanto a experiencias concretas se resaltan algunos esfuerzos colectivos, complementarios a ECOMUN, en materia de reincorporación económica como, por ejemplo:

Frutos de Paz, experiencia de trabajo colectivo en diferentes municipios de Cundinamarca con mercados itinerantes, tiendas urbanas y centros de acopio, apostándole con esto a la descentralización de las relaciones campesino-consumidor final en la cadena de comercialización. A través de Frutos de Paz se potencia el trabajo colectivo que logrará cambiar las vidas tanto de personas firmantes del Acuerdo como de campesinos en los distintos territorios.

AGROPAZ, iniciativa de producción y organización que tiene como fundamento ampliar la base social de participantes con población campesina, víctima, indígena y afro, además de excombatientes. Se creó en el año 2018 y para el 2020 contaba con más de 800 personas vinculadas a la cooperativa, con un $60 \%$ aproximado de integrantes no excombatientes. 
Experiencias como estas y otras existentes, revelan de qué manera se pueden romper estigmatizaciones, desconfianzas y señalamientos, reforzados desde hace tiempo por los medios de comunicación y sectores sociales que se resisten al proceso de reincorporación y al proceso de paz mismo. A partir de un trabajo mancomunado, orientado a superar problemas y necesidades de los territorios, se van logrando también transformaciones en las relaciones y comprensiones sobre la reincorporación. Ese es un trabajo que vincula lo productivo con la construcción de condiciones para la reconciliación.

En relación con este último planteamiento, la asistencia técnica o asesoría general a los proyectos productivos no puede desconocer los elementos necesarios para contribuir a su integralidad y a una concepción que vincule lo productivo con lo relacional y lo social. Quedó en evidencia también la necesidad de asesoría técnica y especial atención al tema de la comercialización, como garantía de sostenibilidad y proyección económica.

\section{Eje 7. Fortalecimiento de capacidades de las y los firmantes del Acuerdo}

"Hemos insistido que la educación se vea como el proyecto más importante para la construcción de paz"11.

Los indicadores institucionales dan cuenta de la gestión gubernamental en materia de educación: más de 5700 personas vinculadas a programas de formación académica, 1400 en primaria, 4200 en bachillerato y alrededor de 180 en otros niveles. La cobertura educativa es de $62 \%$ fuera de los AETCR. De las personas registradas por la ARN, el $86 \%$ han manifestado interés en continuar su proceso educativo: el $24 \%$ en estudios universitarios y el $4 \%$ en estudios de posgrado. Desafortunadamente, desde la representación del componente FARC en el CNR, y desde otros

11 Emilio Archila, conversatorio agosto 12 de 2020. 
sectores de exintegrantes de las FARC-EP, no se percibe una estrategia consolidada para lograrlo.

Desde una postura crítica, se insiste en la necesidad de un Plan Nacional de Educación que permita a ciudadanas y ciudadanos en proceso de reincorporación actuar como sujetos con derechos y obtener los conocimientos necesarios para aportar a la construcción de paz en el país desde distintos frentes. Aquí aparece como consideración importante la posibilidad de homologar saberes y capacidades adquiridas por los y las excombatientes en tiempos de la guerra. Hasta el momento se han logrado algunas certificaciones, pero se han dejado de lado muchos conocimientos que no se recogen en ellas. De otra parte, es importante insistir en el acceso a la educación superior, pues lo logrado en este terreno ha sido a partir de la propia búsqueda y esfuerzo de las personas en reincorporación, y no por iniciativas o programas especiales de admisión en las universidades. En medio de ello se ha contado con el apoyo en este tema de algunas universidades privadas $\mathrm{y}$, en menor cantidad, de algunas públicas como la Universidad Nacional de Colombia.

Las personas en proceso de reincorporación señalan la continuación de la lucha por el acceso a la educación en todos sus niveles, concibiéndola como un proceso que aporta a la transformación personal, colectiva y social. De otra parte, se insiste en pensar el tema educativo más allá de la cobertura con educación formal, para profundizar en las denominadas pedagogías para la paz y, desde ellas, promover procesos de reconciliación y construcción de paz en los territorios.

Se espera que el encuentro entre firmantes del Acuerdo y la institucionalidad, organizado por la Universidad Nacional de Colombia, redunde en avances significativos en el tema educativo, productivo, técnico, social y en una línea poco nombrada pero relevante: el apoyo a la constitución de nuevos núcleos familiares y la proyección de sus hijos e hijas como la 
generación de la paz. Sería necesario, en relación con el tema educativo, identificar con mucha claridad las aspiraciones, expectativas e intereses actuales de la población en proceso de reincorporación.

\section{Eje 8. Incorporación política}

"Ese sujeto que venía desarrollando una acción política desde las armas, ahora desarrolla una acción política en el marco de la normatividad vigente... de la democracia...

Pero en este contexto, el partido no puede ser el único representante legal de la reincorporación"12.

Como avance, éxito o muestra de la reincorporación política, sobresale la creación y consolidación del Partido Comunes, el cual cuenta con personería jurídica y representación parlamentaria a través de nueve curules en el Congreso de la República. Además de ello, en las elecciones de octubre de 2019 fueron elegidos doce candidatos, entre quienes se encuentran tres alcaldes municipales.

De otra parte, se destacan también en relación con este eje temático espacios de concertación como el CNR, con más de 111 sesiones llevadas a cabo; los 24 Consejos Territoriales de Reincorporación y las 12 mesas departamentales de reincorporación, como parte de la infraestructura que visibiliza el proceso adelantado hasta ahora en el escenario político.

La participación política, en un sentido más local/territorial, se lee a la luz de las distintas experiencias organizativas conformadas por personas en reincorporación, algo que va mucho más allá de los procesos electorales. Si bien las asociaciones o cooperativas se van construyendo alrededor de iniciativas de carácter productivo, configuran también escenarios de reivindicación y proyección política para los firmantes del

12 Corpo-Reconciliación, agosto 11 de 2020. 
Acuerdo. Ejemplo de ello es, nuevamente, Frutos de Paz, que como proyecto vinculado a Corpo-Reconciliación no solo es una plataforma para la comercialización de productos desarrollados en las regiones, sino que plantea líneas discursivas y políticas claras desde las economías circulares y los mercados locales. Otras iniciativas como la Cooperativa Agropaz o Asocunt también han venido configurando alianzas con capacidad de convocatoria cada vez mayor. De esta manera su trabajo se orienta a otros ejes como los derechos humanos, vivienda, salud, víctimas, género, etc., a la vez que se genera interlocución con actores institucionales tanto nacionales como internacionales.

En una lógica mucho más vinculada a la vida cotidiana en los territorios, se encuentra la participación política a través de procesos e instancias de organización comunitaria, como las Juntas de Acción Comunal, una de las iniciativas con mayor acogida de parte de la población en reincorporación. Esta articulación con procesos comunitarios locales reviste especial importancia por cuanto posibilitará, a futuro, una mayor articulación territorial y el vínculo real de la población, antes afectada por las acciones de la exguerrilla, con el proceso de reincorporación y con la construcción de paz en el país. Este proceso exige un cambio en la percepción sobre quienes optaron por el uso de las armas para transformar el país y hoy intentan abrirse espacio y tejer relacionamientos de una manera diferente.

Finalmente, en relación con el tema de la incorporación política, queda planteado el reto de construir internamente, como partido, y a través de experiencias conexas, un proceso de unidad en medio de la diversidad y la diferencia. Esto pondrá a prueba la capacidad interna de quienes decidieron dejar las armas. Se espera que de esta experiencia salgan otras claves para la transformación de conflictos al interior de una organización y proceso político. 


\section{Eje 9. Nuevas dinámicas familiares, comunitarias y colectivas}

"No hay modelos que atiendan a comunidades e hijos e hijas de excombatientes (...) La mujer se empoderó en muchos aspectos frente a organización, participación política, salud"13.

Este apartado final comienza con dos frases textuales, aparentemente desarticuladas, pronunciadas en uno de los conversatorios por una mujer integrante de las antiguas FARC-EP. Fueron las dos únicas referencias explícitas a las nuevas dinámicas cotidianas que han ido apareciendo en el tránsito a la vida civil: las relaciones de pareja estables, la maternidad, la paternidad, la constitución de familias, la organización de una vivienda, la construcción de formas de relacionamiento novedosas en medio de un contexto completamente distinto al de la vida cotidiana en la guerra; y asuntos como las relaciones de género, la identidad, la sexualidad, la salud sexual y reproductiva, los cambios en la constitución subjetiva y el proyecto de vida. Todo ello merece especial atención cuando se trata de analizar un proceso de tránsito como la reincorporación. Sobre estos aspectos también debe incluirse una mirada y acción explícita dentro de la política del proceso, porque de lo planteado en los conversatorios casi nada se refiere a ello. Como decía otra excombatiente: "Somos seres humanos...".

En relación con este eje, como Universidad Nacional de Colombia tenemos mucho para aportar. Fundamentalmente a través de espacios que permitan la autorreflexión y la reflexión colectiva de estos aspectos, el análisis de lo que hombres y mujeres quieren para sus vidas, para su futuro; sobre el tipo de familias que quieren construir, el tipo de relaciones de pareja que quieren tener o el tipo de vida que quieren llevar:

13 Dilia Consuelo Fuertes, conversatorio agosto 1 de 2020. 
sus sueños, aspiraciones, etc. Como siempre, el reto está en no perder de vista que todos estos asuntos son también políticos.

\section{Algunas propuestas a manera de conclusiones.}

"La academia no logra entender realmente cuáles son los retos de este proceso de reincorporación"14.

Como Universidad Nacional de Colombia hemos respaldado los procesos conducentes a lo que se conoce como "hacer la paz", asociados a la negociación con las insurgencias específicamente, también denominada 'paz negativa'. Pero nuestro aporte institucional se ha orientado también hacia el sostenimiento de la paz y fundamentalmente hacia su construcción, aportando a la consolidación de un proceso de reincorporación integral, que fortalezca las capacidades para la paz y la transformación de conflictos tanto en las comunidades de los territorios afectados por la guerra como en los colectivos y actores involucrados.

En este sentido, la Universidad Nacional de Colombia decidió constituir la Red de Ética, Cultura, Creación, Ciencia, Tecnología e Innovación para la Paz - Red Paz-, con el fin de coordinar y potenciar las diversas iniciativas y proyectos de aporte a la construcción de paz desarrollados por la institución en sus campos misionales de extensión, investigación y docencia.

Es claro que una prioridad se encuentra en la necesidad de orientar esfuerzos por la reivindicación del derecho a la vida y a la protección tanto de las personas en proceso de reincorporación como de los y las líderes sociales, como un deber del Estado encaminado a superar los factores que inciden en la persistencia de conflictos en diferentes territorios.

Un segundo elemento central es el que representa la importancia de la confluencia de esfuerzos para potenciar los resultados positivos, para

14 Aporte de profesora participante en el conversatorio del $1^{\circ}$ de agosto de 2020. 
que los territorios que han sido el escenario central del conflicto puedan replantear sus perspectivas de desarrollo, recuperando una senda participativa y de cara a los retos de sostenibilidad ambiental.

Finalmente, la recomposición del tejido social y la creación de condiciones para la paz estable y duradera se encuentran en la implementación integral de los acuerdos, con énfasis en la reforma rural integral, en el marco de la cual pueda anidar una cultura de reconciliación fuerte, aspectos en los cuales la Universidad Nacional se compromete a contribuir activamente desde sus capacidades.

\section{Referencias}

Agencia para la Reincorporación y la Normalización. (17 de febrero de 2021). ARN en cifras. Recuperado el 25 de febrero de 2021 de http://www.reincorporacion.gov.co/es/agencia/Paginas/ARN-en-cifras.aspx

Galtung, Johan. (2003). Paz por medios pacíficos: paz y conflicto, desarrollo y civilización. Bilbao: Bakeaz.

Misión de Verificación de las Naciones Unidas en Colombia. (29 de diciembre de 2020). Informe del Secretario General S/2020/1301. Recuperado el 28 de enero de 2021 de https://colombia.unmissions.org/sites/default/files/ sp_n2037704.pdf

Naranjo, Sandra; Machuca, Diana y Valencia, Marcela. (2020). La reforma rural integral en deuda. Bogotá: Gentes del Común/ Centro de Pensamiento y Diálogo Político- CEPDIPO. Recuperado el 30 de enero de 2021 de https://cepdipo.org/portfolio/ cuadernos-de-la-implementacion-6-la-reforma-rural-integral-en-deuda/

Zambrano, Liliana. (2019). La reincorporación colectiva de las FARC-EP: una apuesta estratégica en un entorno adverso. Revista CIDOB D'Afers Internacionals, (121), 45-66. Recuperado el 5 de febrero de 2021 de https://www. jstor.org/stable/26731291 